\title{
Seraphs and Rain
}

\author{
It's been said that angels kill when jealous of love \\ And so I fear what they'll do to us \\ But I will not hide my love for you \\ Sometimes drizzles fall in love with hurricanes \\ But if we were rain, \\ We would fall just the same
}

Michael Voulgarellis 